\title{
DEL ORDEN AL CAOS
}

\section{FROM ORDER TO CHAOS}

\author{
Abril USCANGA BARRADAs**
}

\section{Resumen:}

Regularmente consideramos al derecho como sinónimo de orden, como un instrumento de paz, de justicia, rectitud o equilibrio social, dejando de lado el elemento caótico, ¿será que el derecho también es caos? Estemos o no de acuerdo con la naturaleza misma del derecho, es innegable considerar que el derecho es dinámico y evolutivo, con un necesario carácter social inherente que determina y contiene moral, valores, principios y directrices que guían a una sociedad para determinar qué conductas se adecuan y cuáles se desvían de la obligación establecida en el ordenamiento jurídico. En general, el presente documento pretende mostrar al lector las implicaciones que la dualidad orden y caos simultáneo tienen sobre el derecho.

\section{Palabras clave:}

Caos; contrapuestos; derecho; orden; sistema jurídico.

\begin{abstract}
:
We regularly consider the law as synonymous with order, as an instrument of peace, justice, rectitude or social balance, setting aside the chaotic element. Whether or not we agree with the very nature of law, it is undeniable to consider that Law is dynamic and evolving, with a necessary inherent social character that determines and contains morals, values, principles and guidelines to conduct a society, in order to determine which behaviors are appro-

* Artículo recibido el 12 de agosto de 2019 y aceptado para su publicación el 20 de diciembre de 2019.

${ }^{* *}$ Profesora de tiempo completo, Facultad de Derecho de la UNAM, auscangab@ derecho.unam.mx.
\end{abstract}

Problema. Anuario de Filosofía y Teoría del Derecho, núm. 14, enero-diciembre de 2020, pp. 345-368 Ciudad de México, ISSN 2007-4387, se distribuye bajo una Licencia Creative Commons Reconocimiento-No Comercial-Sin Derivados 4.0 Internacional (CC BY-NC-ND 4.0).

Universidad Nacional Autónoma de México, IIJ-BJV, 2020 


\section{ABRIL USCANGA BARRADAS}

priate and which deviate from the obligation established in the legal system. In general, this document aims to show the reader the implications that the duality of order and simultaneous chaos have on the Law.

Keywords:

Chaos; Opposition; Law; Order; Legal System. 
Sumario: I. Introducción. II. El pensamiento como binomio de contrapuestos. III. El derecho como sistema ordenado. IV. El caos como elemento del derecho. V. Conclusiones. VI. Bibliografía.

\section{INTRODUCCIÓN}

Tradicionalmente, decir derecho es sinónimo de decir orden, pues los diferentes estadios que ha pasado la historia jurídica han estado presididos, en mayor o en menor medida, por el entendido de que el derecho es algo que confiere orden, rectitud y equilibrio en la vida social, de tal manera que se llega a pensar que el derecho, en cuanto tal, es un instrumento absolutamente necesario en la vida de la colectividad, sin el cual se daría lugar a un estado de inestabilidad, desorden, alteración y desorganización de la sociedad.

Las interpretaciones del derecho han variado enormemente a lo largo de los siglos, provocando así discusiones acerca de si éste viene dado por la naturaleza en sí -iusnaturalismo-, por la aspiración del hombre de regular los comportamientos de los gobernados a través de enunciados normativos de deber ser -iuspositivismo-, o por la conceptualización que identifica al derecho con la eficacia normativa asociada a las decisiones judiciales —realismo jurídico-, entre muchas otras formas. ${ }^{1}$

Más allá de discusiones y debates doctrinales acerca de la naturaleza del derecho, lo que resulta innegable y se encuentra fuera de toda duda es que el derecho es dinámico y evolutivo, con un necesario carácter social inherente que determina y contiene la moral, los valores, los principios y directrices que guían a una sociedad, para determinar qué conductas se adecuan y cuáles se desvían de la obligación establecida en el ordenamiento jurídico y, como consecuencia, podrán ameritar un castigo o una sanción; sin embargo, en esta

1 Carnelutti, F., Metodología del derecho, Bogotá, Leyer, 2006, pp. 14 y 15; Vallarta Marrón, José Luis, Introducción al estudio del derecho, Ciudad de México, Porrúa, 2016, p. 41. 
investigación partiremos de la hipótesis que indica que el derecho es mucho más que eso, como así se ha demostrado con la evolución filosófica del pensamiento y, más concretamente, con las interpretaciones del derecho como algo que va más allá de un simple conjunto de normas o de un sistema que regula la sociedad como un todo organizado, resultando que Rivaya ${ }^{2}$ o $\operatorname{Scott}^{3}$ lleguen incluso a plantear al derecho como un elemento que puede encerrar un caos en sí mismo. ${ }^{4}$

El análisis que aquí se ofrece se realiza desde un enfoque inductivo que permite al lector estudiar las implicaciones que la dualidad orden y caos simultáneo tienen sobre el derecho, las cuales, en muchas ocasiones, no se encuentran visibles, por lo que se buscará evidenciar aquellas cuestiones que generalmente pasan desapercibidas a la hora de realizar el análisis del fenómeno jurídico, pero que, a modo de desdoblamiento dual, se desprende del derecho en sí.

De esta forma, el marco teórico se presenta desde un análisis de la filosofía del derecho como binomio de contrapuestos. Para generar este trabajo se consideró la necesidad de una estructura e interconexión sintética entre las ciencias naturales y la ciencia jurídica, así como el estudio de las concepciones evolucionistas del derecho, tanto respecto a ontología del derecho como con la metodología explicativa de los fenómenos jurídicos contemporáneos, para así lograr entender el concepto de orden y caos desde la perspectiva de autores como Thomas Kuhn, Edward Lorenz, Robert Scott, Robert Nozick, Nicklas Luhmann, o Georges Balandier, entre muchos otros teóricos.

\section{EL PENSAMIENTO COMO BINOMIO DE CONTRAPUESTOS}

El hecho de que existan postulados que, como ya se mencionó con anterioridad, abogan por un carácter dual del derecho, hace que sea necesario, en primer término, delimitar lo que se debe entender por orden, para, a continuación, definir lo que es el caos; ello como el es-

2 Rivaya, Benjamín, "Anarquismo y derecho", Revista de Estudios Políticos, Madrid, núm. 112, abril-junio de 2001, p. 78.

3 Scott, Robert E., "Chaos Theory and the Justice Paradox", William \& Mary Law Review, vol. 35, issue I, 1993, pp. 348-351.

4 Rivaya, Benjamín, “Anarquismo y derecho”, cit., p. 78. 
tudio de los contrapuestos que se requieren como binomio para poder valorar la naturaleza del derecho y su estructuración, lo cual simultáneamente pondrá de manifiesto las características principales de aquél, y, en consecuencia, las implicaciones tanto sociales como jurídicas de comprender al derecho como un sistema ordenado, o al caos como un elemento del propio derecho, de tal suerte que se entiende que existe una conexión necesaria entre ciertos periodos de calma y otros de agitación que la ciencia requiere para el rompimiento de paradigmas. ${ }^{5}$

\section{El orden}

Desde la antigua Grecia, Aristóteles había conceptualizado al orden como algo que nos rodea, siendo que el mundo que percibimos con nuestros sentidos está compuesto por toda una serie de cosas que existen para un fin, de tal manera que el orden es algo inherente a la propia naturaleza. ${ }^{6}$ En este sentido, Lozano señala que

...para Aristóteles hay un orden propio del mundo constituido por el movimiento organizado de los astros en el cielo y por el movimiento ordenado de la naturaleza en tanto que suma de la acción regular de los entes individuales. Todos los componentes particulares se mueven o cambian con un único fin, dirigidos por su forma buscan lo mejor para ellos, tienden a su orden particular pero contribuyen entonces a la permanencia del orden del conjunto del que forman parte. ${ }^{7}$

5 De esta forma, se busca romper el paradigma del derecho como un constante orden, ya que incluso Thomas Kuhn describe un modelo de la ciencia basado en periodos de calma, que él llama ciencia normal, en los que reina un determinado paradigma; estos periodos de calma se ven interrumpidos cuando aparecen anomalías en dicho paradigma — respuestas que no pueden ser conseguidas, o resultados y observaciones que contradicen alguno o varios de los principios que sostienen el paradigma-, en estas fases de crisis y agitación es cuando él asegura que se producen las revoluciones científicas que cambian el desarrollo de la ciencia. Véase Kuhn, Thomas, La estructura de las revoluciones científicas, trad. de Agustín Contin, México, Fondo de Cultura Económica, 1971, pp. 27 y 28.

6 Vallarta Marrón, José Luis, Introducción al estudio del derecho, cit., p. 37.

7 Lozano, Vicente, El orden del mundo y su explicación analógica en Aristóteles, España, Espíritu Liv, 2005, p. 334. 
En vista de lo anterior, se puede apreciar que Aristóteles parte de una idea de naturaleza como motor de orden en sí, que sistematiza la realidad, a pesar de encontrarse sujeta a cambios, toda vez que tienen una intención o finalidad específica, concreta y determinada por la propia naturaleza. Es así que cuando Lozano analiza la postura de Aristóteles, menciona que "todos los elementos del mundo sensible que existen por sí mismos... son sustancias naturales que son guiadas por su propia naturaleza o principio inmanente para mantener el orden o naturaleza en el sentido trascendente", 8 por lo que se parte de una existencia de un elemento natural en las cosas u objetos comprendidos en el sentido más lato del término, ${ }^{9}$ incluyéndose dentro de éste, también, a las personas.

Aristóteles afirma que "igual que en los animales, también en la naturaleza había un entendimiento que era la causa del mundo y del orden todo", ${ }^{10}$ con lo que evidencia aún más el hecho de que la naturaleza es el orden en sí, haciendo del orden algo tan esencial y, en palabras del propio Aristóteles, bello, que incluso engloba dentro de lo entendible como bello al mismo orden, a la simetría y a la delimitación. ${ }^{11}$

Este planteamiento ideológico de comprender al mundo en sí como un elemento ordenado, en el que todo lo que existe tiene una finalidad establecida, hace surgir un pensamiento de designio inevitable, en el que la naturaleza tiene propósitos prefijados para cada cosa, para cada persona y para cada elemento, resultando, por ende, en un idealismo tendente a la existencia de un orden rector supremo. ${ }^{12}$

8 Ibidem, p. 335.

9 García Máynez, Eduardo, Filosofía del derecho, México, Porrúa, 1989, pp. 23 y 24.

10 Aristóteles, Metafísica, trad. de Valentín García Yebra, libro I, capítulo 3, 980b 15, p. 6, disponible en: http://cmap.upb.edu.co/rid=1GM19TDTV-2QXBB0-V3Y/Aris toteles\%20-\%20Metafisica.pdf, [20-marzo-2019].

11 Ibidem, libro XIII, capítulo 3, 1078a, 35, p. 125.

12 Este conocimiento empírico de la realidad hace que Cicerón, en su obra Del Hado, reflexione sobre el papel del destino como elemento inevitable en la vida de la comunidad, siendo así que pone de manifiesto que, tras la desaparición de César, se buscaban todos los pretextos de nuevas perturbaciones, por lo que el propio Cicerón debate con su amigo Hircio sobre aquellas medidas que contribuyeran a la paz y a la concordia de los ciudadanos, de tal manera que en el desarrollo del debate surge el planteamiento acerca de si el destino se encuentra prefijado y, por ende, 
Con el pensamiento cristiano se dieron nuevas explicaciones del orden por filósofos como san Agustín de Hipona y santo Tomás de Aquino.

Por lo que respecta al primero de ellos, esto es, san Agustín, presenta, a través de su obra titulada La ciudad de Dios, la situación de dos ciudades como dos realidades metafísicas, "cuya separación empírica, sensible, queda reservada al juicio final de Dios".13 En esta tesitura, el orden se encuentra marcado por lo divino, de tal manera que la dualidad de ciudades hace referencia, por una parte, a una ciudad de Dios, presidida por una felicidad eterna y, por otra, a una ciudad humana, terrestre, en la que reina una infelicidad igualmente eterna. ${ }^{14}$

Así pues, para san Agustín la idea de orden parte de Dios, de modo tal que todo aquello en lo que Dios no interviene no puede ser susceptible de ser considerado orden; así lo establece al afirmar "que todos los reyes y reinos están dispuestos y ordenados por el decreto y potestad del verdadero Dios". ${ }^{15}$ De lo anterior se colige que en tanto en la ciudad de Dios reina el orden y el amor, en contraposición, en la ciudad terrestre, dominada por el hombre, está presente el caos, los apetitos y desórdenes terrenos, ${ }^{16}$ por lo que, en este entendido, para san Agustín, Dios es orden y destino. ${ }^{17}$

Por otra parte, santo Tomás de Aquino continúa el planteamiento del orden preestablecido integrando un marcado matiz dado por la Edad Media, pues vincula el orden al hecho de que existe Dios. Los postulados de Aquino hicieron que Weber le definiera como el genio del orden, ${ }^{18}$ y es que, desde el momento en que Aquino publica su

resultan intrascendentes los actos que se puedan llevar a cabo, toda vez que, de una u otra forma, la tela de la vida ya está tejida y, en consecuencia, no resulta posible esquivar el fatum. Véase Cicerón, Marco Tulio, Del Hado, México, UNAM, 2005, p. 2.

13 San Agustín, La ciudad de Dios, "Introducción", p. 11, disponible en: http://efrue da.com/wp-content/uploads/2011/12/L-a-ciudad-de-Dios.pdf, [20-marzo-2019].

14 Idem.

15 Ibidem, cap. XXXIII, libro cuarto, p. 139.

16 Ibidem, cap. III, libro octavo, p. 219.

17 Ibidem, cap. I, libro quinto, p. 140.

18 Weber, P. J., Santo Tomás de Aquino. El genio del orden, Buenos Aires, Poblet, 1949. 
Summa Theologiae tiene una especial preocupación por conseguir justificar que todos los objetos se encuentran en un orden, ${ }^{19}$ que en el caso de Aquino viene dado por Dios, de tal forma que, en sus propias palabras, "no se nos propone para crear nada si no es en orden a Dios". ${ }^{20}$

Aquino, por lo tanto, si bien considera que existe un orden que preside la existencia de los objetos, éste se encuentra más vinculado con lo establecido por Dios que por una naturaleza en sí del objeto, por lo que si bien continúa en cierto sentido con el planteamiento aristotélico plasmado en su obra Metafísica, ${ }^{21}$ en tanto parte de la existencia de un orden natural, sin embargo, añade ese componente de divinidad en la fórmula creadora del orden.

Continuando con las nociones de orden, el filósofo español Francisco Suárez también presenta una conceptualización del orden en su obra De legibus, en el que se postula a la conciencia humana del mandato divino que obliga al hombre a guardar el orden natural y no perturbarlo. ${ }^{22}$ De esta manera, el orden humano es una implicación o consecuencia intrínseca del orden divino, obteniéndose como conclusión que existe orden humano en tanto la actuación designada por Dios es seguida a modo de mandato expreso y de formal obligatoriedad preceptiva. ${ }^{23}$

Por su parte, García Máynez considera que el orden es "el sometimiento de un conjunto de objetos a una regla o sistema de reglas cuya aplicación hace surgir, entre dichos objetos, las relaciones que permiten realizar las finalidades del ordinante". ${ }^{24}$ Esta definición implica que se eliminen los factores de un Dios, de una ley divina

19 Aquino, Tomás de, Suma teológica, segunda sección, 2a. parte, pp. 11-17, disponible en: http://efrueda.com/wp-content/uploads/2011/12/suma_teologica_2_2. pdf [20-marzo-2019].

20 Ibidem, p. 20.

21 Beuchot, Mauricio, "La metafísica de las causas en Aristóteles y santo Tomás", Logos, vol. IX, núm. 26, 1981, p. 9.

22 Suárez, Francisco, De legibus: De lege naturali, Corpus Hispanorum de Pace, Madrid, Consejo Superior de Investigaciones Científicas, 1974, p. LVII.

${ }^{23}$ Ibidem, p. LVIII.

24 García Máynez, Eduardo, Filosofía del derecho, cit., p. 23. 
o de una fuerza invisible, pero notable, que preside las relaciones entre los sujetos.

En este sentido, Máynez indica cinco elementos esenciales que debe tener todo orden, los cuales son: i) la existencia de un conjunto de objetos; ii) la existencia de una pauta ordenadora; iii) la sujeción de los objetos a la pauta; iv) las relaciones que se derivan para los objetos con motivo de esa sujeción a la pauta, y, v) la finalidad perseguida por el ordinante. ${ }^{25}$

Máynez consideró que la palabra "orden" encierra "dos sentidos que conviene distinguir con pulcritud. En el primero suele aplicarse a las reglas constitutivas del criterio ordenador; en el segundo, al resultado del proceso de ordenación". ${ }^{26}$ Así, se distingue que si bien se está hablando de un mismo término, éste se encuentra integrado por dos planos, que podrían definirse como uno principal y uno derivado, toda vez que al abordar la perspectiva de las reglas constitutivas del propio criterio será necesario atender a todo aquello que pueda llegar a afectar en el ordenador mismo, de igual forma que el resultado de la ordenación dependerá, necesariamente, del criterio utilizado para ordenar.

En resumen, podemos mencionar que el orden se reconoce en diferentes campos como el arte, la axiología, o el propio derecho, considerando igualmente que existen diferentes clasificaciones de orden, como el orden convencional o voluntario, ${ }^{27}$ el orden técnico, ${ }^{28}$ el orden normativo, el orden humano, ${ }^{29} \mathrm{o}$ el orden no humano, pero al mismo tiempo debemos reconocer a contrario sensu la existencia de los contrapuestos, de tal forma que en contraposición al bien se

25 Idem.

${ }^{26}$ Ibidem, p. 26.

27 El criterio convencional o voluntario es el criterio de ordenación creado por un sujeto. Así se establece en García Máynez, Eduardo, Filosofía del derecho, cit., p. 33.

28 Según García Máynez, se da este nombre a los órdenes cuya finalidad derivan de la aplicación de reglas de una o más artes y son contrapuestos a aquellos cuyos fines que se logran a través del cumplimiento de normas. García Máynez, Eduardo, Filosofía del derecho, cit., 35.

29 En este caso no nos referimos a algo establecido cognoscible y utilizable, sino a una tarea, objetivo o propósito del hombre en sí. Kuhn, Helmut, El Estado. Una exposición filosófica, Madrid, Rialp, 1979, p. 19. 
encuentra el mal, en contraposición a lo árido está lo fértil, y como elemento antagónico del orden se encuentra el caos.

\section{El caos}

Del mismo modo que, como ya se ha visto, el orden ha sido objeto de estudio por diferentes autores, el caos ha sido igualmente analizado, por lo que a pesar de que al hablar de éste se genera en el oyente un pensamiento automático de desbarajuste, desorden ${ }^{30} \mathrm{o}$ desorganización, sin embargo, resulta de mucho interés valorarlo y ver su naturaleza, características y aplicación en el derecho.

El hecho de apartarse de un orden determinado con carácter previo implica provocar un fuerte cambio en el paradigma establecido; no obstante, el hecho de que se modifique un pensamiento instaurado y aceptado por la generalidad de la sociedad en un momento determinado no supone, en absoluto, que dicha transformación no entrañe un importante interés, pues se llega incluso a postular al desorden como un sistema que, aunque quizá parezca una idea poco ortodoxa, puede llegar a implementarse de tal manera que sea aquél el que pueda regir la vida de la sociedad, y es que, como se colige de las palabras del gran científico alemán Albert Einstein, en el desorden se encuentra la simplicidad. ${ }^{31}$

El caos, al igual que sucede con el orden, es un término multívoco, que por tanto acepta muchas y muy diferentes interpretaciones o conceptualizaciones. ${ }^{32}$ En este sentido, García Meza indica que el caos puede llegar a entenderse desde comportamientos aparentemente recurrentes y aleatorios en un sistema determinístico simple, hasta cierto tipo de orden sin periodicidad, sin olvidar su posible conceptualización como dinámica de sistemas liberados y de la pre-

30 Eduardo Alejandro Ibáñez señala que es muy importante alejarse de la idea de imprevisibilidad absoluta que el término desorden puede hacer nacer en la mente del individuo, pues sería más correcto hablar de seudo aleatoriedad. Bunge, Mario, Diccionario de filosofía, México, Siglo XXI, 2001, p. 19.

31 Levine, Michael, 7 Life Lessons from Noah's Ark: How to Survive a Flood in Your Own Life, Berkeley, Celestial Arts, 2003, p. 25.

32 Gleick, James, Chaos. Making a New Science, Ontario, Penguin Books, 1987. 
dictibilidad para explorar aleatoriamente cada una de sus posibilidades dinámicas. ${ }^{33}$

Resulta importante diferenciar la teoría del caos en sus dos vertientes, pues, por una parte, se encuentra la teoría del caos estocástico y, por otra, está la teoría del caos determinista. ${ }^{34}$ Una y otra guardan similitudes, sin embargo no deben confundirse, ya que la primera de ellas - caos estocástico- es totalmente imprevisible, aleatoria e impredecible, mientras que la segunda - caos determinista- se ocupa del estudio de ciertos sistemas de naturaleza dinámica, esto es, modificable y evolutiva, que resultan ser sensibles a las variaciones de las condiciones iniciales del propio sistema, haciendo que las más mínimas variaciones se traduzcan en grandes consecuencias en el futuro, por lo cual la predicción a largo plazo resulta realmente compleja. ${ }^{35}$

La generación de la teoría del caos como tal se planteó y, posteriormente, se fue perfeccionando con el paso de los años, siendo que uno de los momentos incipientes de esta teoría fue proporcionado por Georg Cantor a finales del siglo XIX y principios del siglo XX en el área matemática, al plantear la posibilidad de dividir intervalos unitarios de manera infinita. ${ }^{36}$ Como continuación del planteamiento de Cantor en cuanto a teoría de un caos existente se refiere, adquirieron una especial importancia los pensamientos del matemático francés Henri Poincaré, quien creó los sistemas dinámicos, dotando así de una fuerza notoria y visible a los postulados del caos, pues Poincaré estudió el problema del movimiento de los cuerpos a través de fases. Este postulado establece que a través de los movimientos de los cuerpos se desencadenan efectos impredecibles, con lo que llega a la conclusión de que si el sistema establecido es leve-

${ }^{33}$ García Meza, Carlos Jesús, La teoría de caos: algunas implicaciones en el área de la metodología en la ciencia, Monterrey, Universidad Autónoma de Nuevo León, 1993, p. 11.

34 Ibáñez, Eduardo Alejandro, Las teorías del caos, la complejidad y los sistemas, Santa Fe, Homo Sapiens, 2008, p. 14.

35 Ibidem, p. 15.

36 Dauben, Joseph Warren, Georg Cantor: His Mathematics and Philosophy of the Infinite, Princeton University Press, 1990, pp. 120-149. 
mente perturbado, los efectos de esa mínima perturbación entrañan gran complejidad, ya que impiden o alteran, de una manera amplia, la posibilidad de previsión respecto de los resultados que se puedan llegar a obtener. ${ }^{37}$

Este planteamiento que indica que la más mínima alteración en las condiciones iniciales puede desplegar consecuencias importantes en el resultado será lo que, años más tarde, retome Edward Lorenz para presentar el denominado "efecto mariposa". ${ }^{38} \mathrm{La}$ formulación del efecto mariposa como exponente de la teoría del caos determinista tuvo su origen en un estudio basado en el clima, mediante el cual Lorenz pretendía explicar el motivo que hace que sea tan complicado poder obtener previsiones meteorológicas con cierta seguridad y con una antelación suficiente, llegando a la conclusión de que hasta el aleteo de una mariposa en Brasil puede llegar a provocar un tornado en Texas. ${ }^{39}$

El efecto mariposa resultó ser una extensión del planteamiento de Poincaré; sin embargo, en el perfeccionamiento de la teoría de Lorenz también tuvo su parte de importancia el postulado formulado por Duffing y Van der Pol, quienes siguiendo esa concepción dinámica de lo que nos rodea demostraron que la variación de un parámetro en un estudio podría poner de manifiesto la existencia de toda una secuencia de posibles resultados que serían susceptibles de llegar a provocar un estado caótico. ${ }^{40}$

En esta tesitura, Ibáñez determina que "para un sistema altamente inestable, la más mínima desviación, perturbación, error o divergencia en su estado inicial, se amplificará exponencialmente

37 Gray, Jeremy, Henri Poincaré. A Scientific Biography, Princeton University Press, 2013, pp. 27-34.

38 Lorenz, Edward, "The Butterfly Effect”, en Abraham, Ralph y Ueda, Yoshisuke (eds.), The Chaos Avant-Garde. Memories of the Early Days of Chaos Theory, United Kingdom, World Scientific Publishing, 2000, pp. 91-95.

${ }^{39}$ Al respecto se puede consultar el archivo Lorenz, Edward N., Previsibilidad: ¿debe el aleteo de una mariposa en Brasil originar un tornado en Texas?, trad. de Andrea M. Cristiani, disponible en: https://elartedepreguntar.files.wordpress.com/ 2009/12/edward-lorenz_esp.pdf [12-marzo-2019].

40 Camurri Righi, Antonio, Resonancia no lineal, Concepción, Universidad de Concepción, Consejo de Investigación Científica, 1967, pp. 11-24. 
con el curso del tiempo tornando imposible la previsión de su estado final allende ese lapso". ${ }^{41}$ De esta forma, se pone de manifiesto que "la caoticidad es una propiedad inherente a la propia dinámica interna del sistema", ${ }^{42}$ toda vez que tanto los sistemas simples como los complejos pueden ser caóticos desde el momento en que ese caos proviene de la sensibilidad a las condiciones iniciales; esto es, las interacciones locales del propio sistema, por lo que el número de variables presentes en ese sistema no representan un componente determinante para que surja el caos, pues lo que realmente provoca el caos es, como decimos, que las conductas iniciales o comportamientos primarios tengan una mayor o menor susceptibilidad de afectación en virtud de las circunstancias que rodean al sistema en específico.

Independientemente de si se sigue una teoría o concepción del caos como absoluta imprevisibilidad — caos estocástico-, como si se aboga por un caos en el que subyace un orden con una posibilidad de predicción muy compleja, pero existente, al fin y al cabo - caos determinista - el caos provoca una ruptura o quiebre del orden previamente fijado. Es por ello que el caos, comprendido en este sentido, hace que se pueda llegar a poner en duda el orden previamente establecido en la comunidad social, dotando de apertura al pensamiento científico y filosófico, y es que del caos surgen nuevas estructuras, denominadas "estructuras disipativas", que están alejadas del equilibrio, pero que son coherentes en tanto desarrollan una auto organización. ${ }^{43}$

La aparición de estas estructuras disipativas pone de manifiesto una circunstancia muy importante, y es que aun en el caos existe un orden, no entendido como paradigma establecido de pensamiento o distribución de los objetos, sino como un concierto, serie o modo de realización de las cosas.

41 Ibáñez, Eduardo Alejandro, Las teorías del caos..., cit., p. 15.

42 Ibidem, p. 17.

${ }^{43}$ García Velarde, Manuel, "Estructuras dispositivas. Algunas nociones básicas 1", El Basilisco, núm.10, mayo-octubre de 1980, pp. 8-13. 


\section{EL DERECHO COMO SISTEMA ORDENADO}

El derecho y su finalidad en términos teóricos se puede acercar más a la acepción de orden, pero en la práctica parece alejarse del orden y tener una relación más próxima al caos, por lo que podemos preguntarnos ¿el orden no es orden por el derecho?

García Máynez afirma que el orden jurídico real de la sociedad no es el sistema normativo de ésta, sino el resultado de la sujeción de los destinatarios a las normas; es decir, sólo cuando las normas son cumplidas o aplicadas, el orden jurídico se convierte en realidad, ${ }^{44}$ lo que a contrario sensu supone que si no hay cumplimiento de las normas jurídicas, no existe un orden jurídico como tal, con lo que ya no se podría hablar de orden por derecho.

En este sentido, la palabra "orden" es el leitmotiv que subsiste bajo el entendimiento del derecho, lo cual nos hace preguntarnos si ese orden está preestablecido y si toda consecuencia es resultado de un orden prefijado, lo que llevaría a suponer que en toda realidad existe la posibilidad de que un designio resulte inevitable. ${ }^{45}$

En este sentido, es importante señalar, como lo hace García Máynez, la existencia de dos tipos de orden, que son el orden interno y el orden externo.

El orden interno se refiere a un comportamiento del individuo que se deriva del ejercicio de sus capacidades, por lo que su conducta condiciona la felicidad y virtudes, generando su esencia. ${ }^{46}$ Este orden interno es importante tomarlo en perspectiva con el derecho, pues es éste el que conforma el orden externo en tanto regulación del comportamiento del individuo como elemento restrictivo del orden interno. Así, se puede hablar del derecho como orden porque regula la conducta interna del sujeto, haciendo que se forme una idea conjunta o común respecto del modo en que se debe regir la sociedad, pues, de lo contrario, la persona podría atender única-

44 García Máynez, Eduardo, Filosofía del derecho, cit., p. 20.

45 Este planteamiento puede provocar interesantes debates cuando se valoran las opciones reales de desarrollo o de libertad del individuo por sí mismo.

46 García Máynez, Eduardo, Filosofía del derecho, cit., p. 55. 
mente a su orden interno, esto es, a sus deseos y sus concepciones de lo que es bueno, malo, o de lo que le provoca felicidad, concepciones que pueden llegar a confrontarse directamente con las interpretaciones que otros individuos de la colectividad pudieran llegar a tener respecto de esas categorías, por lo que el derecho como orden externo sirve de limitante a esos deseos internos propiciando un orden como tal en la sociedad.

Esta distinción entre orden interno y orden externo cobra una mayor importancia en el momento en que se atiende de manera escrupulosa al derecho, entendiéndolo ya como algo que ordena la vida colectiva a través de las normas jurídicas, las cuales existen, precisamente, porque existe un ordenamiento que las habilita, y es que, siguiendo las palabras de Bobbio, "la expresión derecho se refiere a un determinado tipo de ordenamiento". ${ }^{7}$

El ordenamiento existe porque existe un orden que debe ser atendido, el cual, para ser respetado, necesita de instrumentos que revistan de fuerza al orden en sí, propiciándose las normas jurídicas. ${ }^{48}$

Ahora bien, es trascendental considerar que si bien existe un orden natural al cual abona el derecho, ${ }^{49}$ sin embargo se debe entender que el orden natural no es un sistema acabado de principios inmodificables y eternos, que funge como modelo de absolutamente todo derecho válido, eficaz, vigente y positivo, ${ }^{50}$ sino que hay que considerar al orden natural como directriz de las situaciones presentes o futuras, por lo que sirve de base para que el derecho se pueda desarrollar atendiendo a las necesidades circunstanciales de cada momento; de lo contrario, implicaría pretender positivizar un derecho absoluto que abarque la totalidad de los supuestos posibles y futuros.

47 Bobbio, Norberto, Teoría general del derecho, 2a. ed., Santa Fé de Bogotá, Temis, 1997, p. 149.

48 Sobre las normas jurídicas, véase García Máynez, Eduardo, Introducción al estudio del derecho, 5a. ed., México, Porrúa, 2002, pp. 78-97.

49 Siguiendo a Carrancá y Rivas, el derecho en su conjunto es una proposición de deber ser. Carrancá y Rivas, Raúl, Filosofía del derecho penal, México, Porrúa, 2009, p. 1.

$50 \quad$ Ibidem, p. 48. 


\section{EL CAOS COMO ELEMENTO DEL DERECHO}

La evolución y, sobre todo, la interpretación del derecho, desde un ámbito teórico o práctico contemporáneo —decisiones judiciales-, ponen sobre la mesa la existencia de un caos inherente a la naturaleza jurídica. Guerrero Olvera indica que hay distintos tipos de caos en el derecho que podemos encontrar en diferentes ámbitos, como el teórico, el de las fuentes y el de la decisión judicial. ${ }^{51}$

La realidad contemporánea evidencia que en el derecho existe tendencia a un caos palpable. Así, podemos mencionar los siguientes ejemplos:

a) El empleo del monopolio jurídico (tribunales) y del orden (policía) en donde no se tiene necesariamente como finalidad el beneficio social. ${ }^{52}$

b) La democracia parece haber agudizado la utilización del derecho como instrumento al servicio de unos pocos, para lograr fines que están presididos por la consecución de metas que parecen orientarse más hacia la particularidad. ${ }^{53}$

c) El caos legislativo, que implica, según Hoppe, que "el número de actos legislativos y reglamentaciones promulgadas por los parlamentos en el transcurso de un año normal está hoy en las decenas de millares, llenando centenares de miles de páginas, afectando a todos los aspectos de la vida civil y comercial, de lo que resulta una constante depreciación de la ley y el aumento de la inseguridad jurídica". ${ }^{54}$

d) La existencia, de acuerdo con Ferrajoli, de un vacío de poder, que permite la existencia de nuevos poderes, que se tra-

51 Guerrero Olvera, Sergio Arturo, "El caos del derecho y el derecho al caos. Reflexiones acerca de la ley de «lo uno o lo otro»", en Pensar el derecho, Querétaro, Fundación Universitaria de Derecho, Administración y Política, 2007, p. 75.

52 Hoppe, Hans-Hermann, Monarquía, democracia y orden natural, Madrid, Unión, 2013, p. 60.

53 Jouvenel, Bertrand de, La soberanía, Granada, Comares, 2016, pp. 334 y 335.

54 Hoppe, Hans-Hermann, Monarquía, democracia y orden natural..., cit., p. 108. 
ducen en poderes fácticos, poderes desregulados y salvajes tanto del mercado como de la política, los cuales toman acción, generándose así una selección guiada por la ley del más fuerte..$^{55}$

e) Para Luhmann, el caos viene dado por la contingencia, la cual se trata, entre otras cosas, del reverso de los valores inviolables. ${ }^{56}$ Este planteamiento hace considerar un elemento que puede romper el orden establecido, siendo que dicho elemento proviene precisamente de la naturaleza, toda vez que "todo suceso siempre tiene lugar en el contexto de la contingencia". ${ }^{7}$

f) El caos incluso se puede generar desde un apartado semántico, provocando así un desorden en lo más básico: su concepto. ${ }^{58}$

g) También podríamos decir que "identificar cuántas y cuáles son las normas jurídicas es una tarea imposible", ${ }^{59}$ o, al menos, compleja, ya que existen reconocimientos diversos en torno a la naturaleza y características que la norma debe contener, obteniéndose como consecuencia que aquellas disposiciones que no cuentan con una serie de notas distintivas específicas previamente establecidas no sean consideradas como normas jurídicas.

Con base en lo anterior, se debe mencionar que el derecho también puede partir del caos, pues, siguiendo el pensamiento de Balandier, en virtud del cual el desorden es innato a la sociedad moderna, se extrae como resultado que el derecho cae en el desorden

55 Ferrajoli, Luigi, "Los retos de la procuración de justicia en un mundo globalizado", Revista de la Facultad de Derecho de México, México, UNAM, vol. 58, núm. 250, 2008, p. 30.

56 Luhmann, Nicklas, "La contingencia como atributo de la sociedad moderna", en Giddens, Anthony et al. (eds.), Las consecuencias perversas de la modernidad. Modernidad, contingencia y riesgo, Barcelona, Anthropos, 1996, p. 174.

57 Ibidem, p. 173.

58 Guerrero Olvera, Sergio Arturo, El caos del derecho y el derecho al caos..., cit., p. 75.

59 Ibidem, p. 77. 
en tanto elemento natural presente en esa misma colectividad actual rigiendo "las formas de negociar la vida cotidiana". 60

El razonamiento que se extrae del pensamiento de Luhmann cobra mayor fuerza con las palabras de Donatien Alphonse François de Sade, más conocido como Marqués de Sade, quien en su obra Juliette 1 sostiene no solamente que el caos o desorden es natural, sino que además ese desorden es el que da lugar al orden, lo cual se produce, también, por la propia naturaleza, ${ }^{61}$ llegando incluso a aseverar que sin desorden no se llegaría al orden. ${ }^{62}$

Este postulado implica que, trasladado al ámbito del derecho en concreto, se debe partir de la idea de un desorden que provoca el orden, por lo que si hablamos, como el título del presente escrito reza, del orden al caos, se puede llegar a un razonamiento en virtud del cual la sociedad contemporánea ha llegado a un punto en el que, tal vez, resulta necesario deconstruir los elementos que componen la estructura conceptual del derecho actual para, a partir de ese caos generado, volver a reconstruir el derecho.

El ámbito de la decisión judicial es en donde, quizá, se aprecia con mayor claridad el desorden o caos presente en el derecho, pues la libre valoración de la prueba, el razonamiento puramente subjetivo respecto del caso sometido a litigio y la interpretación de las normas aplicables a aquél revelan la existencia de una falta de certeza jurídica en el derecho, que debería ser, precisamente, el elemento que dotara de seguridad a la colectividad.

En este sentido, cobran gran importancia las palabras de Ronald Dworkin cuando indica que parte del proceso jurídico consiste en que el juez tome entre sus manos el texto constitucional y lo aplique a situaciones concretas, ${ }^{63}$ pues esa interpretación judicial, en gran medida, adolece de una falta de baremos o niveles medidores respecto de la obtención de una garantía en cuanto a un posible resul-

60 Balandier, Georges, El desorden. La teoría del caos y las ciencias sociales, Barcelona, Gedisa, 2003, p. 173.

61 Sade, Marqués de, Juliette 1, Librodoc, p. 99, disponible en: http://seronoser. free.fr/sade/1800\%20Juliette\%201.pdf [22-marzo-2019].

62 Idem.

63 Dworkin, Ronald, El imperio de la justicia, Barcelona, Gedisa, 1992, p. 233. 
tado, de tal modo que en el derecho no existe una fórmula que establezca que si se realiza A, la consecuencia será B indefectiblemente, sino que más bien habría que hablar de que si se realiza $\mathrm{A}$, las posibles consecuencias pueden ser B, C, D, E, etcetéra, en función de las circunstancias que acontecen en $\mathrm{A}$.

Lo anterior es explicado por Hans Kelsen a través de la teoría de la causalidad y de la imputación, la cual parte de la base de que el derecho se rige por la imputación, mientras que la naturaleza se asienta en la causalidad. De esta manera, en la naturaleza, el físico describe con leyes los fenómenos que existen, pues siempre que se da A, tendrá lugar B; mientras que el jurista, en el derecho, relaciona la conducta del individuo con el enunciado del deber ser establecido en la norma para generar una relación de imputación entre ambos, siendo que si el individuo comete la conducta $\mathrm{A}$, no necesariamente tendrá que producirse la consecuencia $\mathrm{B}$, a diferencia de las leyes naturales, cuyos resultados serán el efecto obligatorio de la causa anterior, los cuales se producen independientemente de toda norma creada por los hombres. ${ }^{64}$

En consecuencia, se puede señalar que el derecho, en su aplicación, puede llegar a determinaciones y criterios encontrados, pues mientras para un mismo caso un operador judicial resuelve $X$, para otro la respuesta puede ser Y, lo que en gran medida es susceptible de representar un caos potencial que implique la indeterminación, discrecionalidad, subjetividad o incertidumbre, haciendo del derecho un sistema dúctil de textura abierta.

El derecho no puede ser entendido e interpretado como un sistema lineal o estructura ideal, sino que cuenta con variables, condicionantes, componentes y aspectos que no pueden ser previstos y, por lo tanto, regulados con exactitud, lo que hace que más allá del orden natural que establece la existencia del derecho, hay, también, un caos en él como consecuencia de esa imprevisibilidad.

${ }^{64}$ Kelsen, Hans, Teoría pura del derecho, trad. de Roberto J. Vernengo, México, UNAM, Instituto de Investigaciones Jurídicas, 2007, pp. 89 y 90. 


\section{CONCLUSIONES}

El derecho en sus diferentes ámbitos puede mostrarnos facetas que reproducen el orden o el caos de forma dinámica, en la medida en que pueden coexistir a través del estudio del origen, contenido y aplicación de un sistema jurídico, procurando que, a través de los antagónicos, podamos dar respuesta o comprender las problemáticas que la ciencia jurídica contiene.

En este sentido, se podría considerar que el caos y el orden son términos mutuamente contradictorios, pero a través del estudio de los contrapuestos consiguen validarse mutuamente como dos factores básicos del binomio jurídico que bien podrían coexistir en un mismo mundo.

Por una parte, podemos decir que las teorías proponen y demuestran que nuestra concepción lineal del orden es excesivamente reductiva y pobre, mientras que las teorías del caos pueden ofrecer a los juristas la posibilidad de comprender la realidad como un todo interdependiente, dar respuestas a lo aparentemente desordenado y entender que el derecho no se agota en las normas, sino que implica un orden integral.

En la práctica podemos percatarnos de que las continuas promulgaciones y modificaciones legislativas o el cuestionado quehacer judicial hacen que se piense en la existencia de un caos en el derecho, surgiendo dudas respecto de si realmente el derecho es orden, pues la falta de certeza jurídica que surge en la sociedad como consecuencia de conductas soberanas del legislador o del operador judicial parecen responder más a un deseo particular que a un bien común, pues dejan, cuanto menos, en entredicho al derecho y generan falta de confianza en él como instrumento de solución de los problemas sociales.

De esta manera, han surgido teóricos como Robert Nozick ${ }^{65}$ que consideran que la eliminación del derecho sería una opción, siguiendo así postulados de corte anarquista. A pesar de ello, se debe destacar que tales planteamientos no serían correctos, ya que la desaparición de aquél traería consigo no sólo la falta de orden, sino

65 Para más información, véase Nozick, Robert, Anarquía, Estado y utopía, México, Fondo de Cultura Económica, 1988. 
el caos total seguido por la ausencia de gobierno y la presencia de una gran desorganización que provocarían el regreso del hombre como auténtico lobo para el hombre ${ }^{66}$ recordando así el latinismo homo homini lupus ${ }^{67}$ establecido por Hobbes.

En sentido contrario, el orden permite el fenómeno de la interacción social, que inicia con la conducta entre dos o más seres humanos que se relacionan y construyen un sistema de orden superior, compuesto por elementos con propiedades constitutivas o emergentes, que son y no son, respectivamente, atribuibles a los individuos que constituyen tal sistema, de forma que podríamos decir que se crean sistemas sociales autopoiéticos. ${ }^{68}$

Aunado a lo anterior, es importante considerar que el caos no es desorden puro en sí mismo, pues del propio caos subyace un orden que hace que los objetos se coloquen en el lugar que les corresponde, disponiéndose entre sí, por lo que se podría llegar a decir que si el orden es natural, el caos lo es igualmente.

Finalmente, podemos concluir que el orden y el caos son elementos que podríamos considerar como necesarios en el derecho, toda vez que a partir de ellos se puede llegar a reconstruir y dar dinamismo al sistema jurídico para reevaluar los conceptos, postulados y enunciados jurídicos fundamentales, que con motivo de ciertos factores o situaciones circunstanciales resultan inoperativos, injustos, ineficientes o inútiles para el derecho. Es así que podemos advertir que, como manifiesta Guerrero Olvera, "tal vez la teoría del caos sea una buena opción para... aceptar el carácter difuso del derecho, o, al menos, una esperanza para comprender mejor el comportamiento de los sistemas jurídicos frente a su función de coordinar las relaciones sociales", 69 pues el derecho cuenta con manifestaciones comple-

66 Hobbes, Thomas, De cive. Elementos filosóficos sobre el ciudadano, trad. de Carlos Mellizo, Madrid, Alianza, 2000, pp. 33 y 34.

67 Hobbes, Thomas, Leviatán o la materia, forma y poder de Estado eclesiástico y civil, trad. de Carlos Mellizo, Madrid, Alianza, 2009, p. IV.

68 Humberto Maturana y Niklas Luhmann llevaron a cabo estudios para analizar la teoría de sistemas y los sistemas autopoiéticos. En este sentido se postuló que la sociedad es un sistema social autopoiético (clausurados operativamente) y autorreferente (que integran los elementos del sistema como unidades de función).

69 Guerrero Olvera, Sergio Arturo, El caos del derecho..., cit., p. 85. 
jas y aleatorias que hacen que no pueda ser estudiado únicamente de una manera lineal.

\section{BIBLIOGRAFÍA}

Aquino, Tomás de, Suma de teología, segunda sección, 2a. parte, disponible en: http://efrueda.com/wp-content/uploads/20 11/12/suma_teologica_2_2.pdf.

ARISTóteles, Metafísica, trad. de Valentín García Yebra, disponible en: http://cmap.upb.edu.co/rid=1GM19TDTV-2QXBB0-V3Y/ Aristoteles\%20-\%20Metafisica.pdf.

BALANDIER, Georges, El desorden. La teoría del caos y las ciencias sociales, Barcelona, Gedisa, 2003.

Beuchot, Mauricio, "La metafísica de las causas en Aristóteles y santo Tomás”, Logos, vol. IX, núm. 26, 1981.

Boвbio, Norberto, Teoría general del derecho, 2a. ed., Santa Fé de Bogotá, Temis, 1997.

Bunge, Mario, Diccionario de filosofía, México, Siglo XXI, 2001.

CAmurri Righi, Antonio, Resonancia no lineal, Concepción, Universidad de Concepción, Consejo de Investigación Científica, 1967.

CARnelutti, F., Metodología del derecho, Bogotá, Leyer, 2006.

CARrancá y Rivas, Raúl, Filosofía del derecho penal, México, Porrúa, 2009.

Cicerón, Marco Tulio, Del Hado, México, UNAM, 2005.

Dauben, Joseph Warren, Georg Cantor: His mathematics and Philosophy of the Infinite, Princeton, Princeton University Press, 1990.

Dworkin, Ronald, El imperio de la justicia, Barcelona, Gedisa, 1992.

FErRAjoli, Luigi, "Los retos de la procuración de justicia en un mundo globalizado", Revista de la Facultad de Derecho de México, México, UNAM, vol. 58, núm. 250, 2008. 
García MáYnez, Eduardo, Filosofía del derecho, México, Porrúa, 1989.

GARCÍA MÁYNEZ, Eduardo, Introducción al estudio del derecho, 5a. ed., México, Porrúa, 2002.

García Meza, Carlos Jesús, La teoría de caos: algunas implicaciones en el área de la metodología en la ciencia, Monterrey, Universidad Autónoma de Nuevo León, 1993.

García Velarde, Manuel, "Estructuras dispositivas. Algunas nociones básicas 1", El Basilisco, núm. 10, mayo-octubre de 1980.

GLEICK, James, Chaos. Making a New Science, Ontario, Penguin Books, 1987.

Gray, Jeremy, Henri Poincaré. A Scientific Biography, Princeton, Princeton University Press, 2013.

Guerrero Olvera, Sergio Arturo, "El caos del derecho y el derecho al caos. Reflexiones acerca de la ley de "lo uno o lo otro»", en Pensar el derecho, Querétaro, Fundación Universitaria de derecho, Administración y Política, 2007.

HobBes, Thomas, De cive. Elementos filosóficos sobre el ciudadano, trad. de Carlos Mellizo, Madrid, Alianza, 2000.

HoвBEs, Thomas, Leviatán o la materia, forma y poder de Estado eclesiástico y civil, trad. de Carlos Mellizo, Madrid, Alianza, 2009.

Hoppe, Hans-Hermann, Monarquía, democracia y orden natural. Una visión austriaca de la era americana, Madrid, Unión, 2013.

IBÁÑEz, Eduardo Alejandro, Las teorías del caos, la complejidad y los sistemas, Santa Fe, Homo Sapiens Ediciones, 2008.

Jouvenel, Bertrand de, La soberanía, Granada, Comares, 2016.

Kelsen, Hans, Teoría pura del derecho, trad. de Roberto J. Vernengo, México, UNAM, Instituto de Investigaciones Jurídicas, 2007.

Kunn, Helmut, El Estado. Una exposición filosófica, Madrid, Rialp, 1979.

KuHN, Thomas, La estructura de las revoluciones científicas, trad. de Agustín Contin, México, Fondo de Cultura Económica, 1971.

LEVInE, Michael, 7 Life Lessons from Noah's Ark: How to Survive a Flood in Your Own Life, Berkeley, Celestial Arts, 2003. 
Lorenz, Edward N., Previsibilidad: ¿debe el aleteo de una mariposa en Brasil originar un tornado en Texas?, trad. de Andrea M. Cristiani, disponible en: https://elartedepreguntar.files.word pre ss.com/2009/12/edward-lorenz_esp.pdf.

LORENZ, Edward, "The butterfly effect", en ABRAHAM, Ralph y UEDA, Yoshisuke (eds.), The Chaos Vant-Garde. Memories of the Early Days of Chaos Theory, United Kingdom, World Scientific Publishing, 2000.

LozANo, Vicente, El orden del mundo y su explicación analógica en Aristóteles, Espíritu Liv, 2005.

LuHMANN, Nicklas, "La contingencia como atributo de la sociedad moderna", en GidDENS, Anthony et al. (eds.), Las consecuencias perversas de la modernidad. Modernidad, contingencia y riesgo, Barcelona, Anthropos, 1996.

Nozick, Robert, Anarquía, Estado y utopía, México, Fondo de Cultura Económica, 1988.

RivaYA, Benjamín, "Anarquismo y derecho", Revista de Estudios Políticos, Madrid, núm. 112, abril-junio de 2001.

SADE, Marqués de, Juliette 1, Librodoc, disponible en: http://serono ser.free.fr/sade/1800\%20Juliette\%201.pdf.

San Agustín, La ciudad de Dios, disponible en: http://efrueda.com/ wp-content/uploads/2011/12/L-a-ciudad-de-Dios.pdf.

ScotT, Robert E., "Chaos Theory and the Justice Paradox", William \& Mary Law Review, vol. 35, issue I, 1993.

SuÁrez, Francisco, De legibus: De lege naturali, Corpus Hispanorum de pace, Madrid, Consejo Superior de Investigaciones Científicas, 1974.

VAllarta Marrón, José Luis, Introducción al estudio del derecho, México, Porrúa, 2016.

Weber, P. J., Santo Tomás de Aquino. El genio del orden, Buenos Aires, Poblet, 1949. 